\title{
Improved Second-Order Balanced Truncation for Symmetric Systems
}

\author{
Peter Benner*,** Patrick Kürschner* Jens Saak*,** \\ * Max Planck Institute for Dynamics of Complex Technical Systems, \\ 39106 Magdeburg, Germany \\ ** Department of Mathematics, TU Chemnitz, 09107 Chemnitz, \\ Germany
}

\begin{abstract}
We investigate second order balanced truncation model order reduction for symmetric linear time invariant second order control systems. This special structure decreases the required computational effort significantly. Moreover, we show how stability of the original model can be preserved for such systems. We briefly discuss the numerical solution of the occurring large-scale Lyapunov equations with a modified low-rank ADI method. The approach is tested on finite element models of mechanical structures.
\end{abstract}

Keywords: model reduction, balanced truncation, second order system, Lyapunov equation

\section{INTRODUCTION}

In this work we consider model reduction for linear, timeinvariant control systems of second order:

$$
\begin{aligned}
M \ddot{x}(t)+D \dot{x}(t)+K x(t) & =B u(t), \\
y(t) & =C^{\mathrm{v}} \dot{x}(t)+C^{\mathrm{p}} x(t),
\end{aligned}
$$

where $M, D, K \in \mathbb{R}^{n \times n}$ are usually referred to as the mass, damping and stiffness matrix, $B \in \mathbb{R}^{n \times m}$ is the input matrix and $C^{\mathrm{p}}, C^{\mathrm{v}} \in \mathbb{R}^{p \times n}$ are position and velocity output matrices. Such systems arise, for instance, in the vibration analysis of elastic mechanical bodies. A common way to model the elastic deformations of such a structure is using the Finite Element method which will introduce a high number of elastic degrees of freedom and thus drastically increase the state space dimension of the model compared to a rigid body model of the structure. In order to carry out time-domain simulations efficiently, this large number of degrees of freedom is reduced by model order reduction approaches.

To obtain accurate reduced order models we use balanced truncation (Moore, 1981) here as model order reduction method which has a system theoretic background. We focus on symmetric second order systems whose properties turn out to be beneficial for our purposes from both a theoretical and computational point of view. Following the second order balanced truncation approach (Reis and Stykel., 2008), the original second order structure can be preserved. For solving the inherent Lyapunov equation, the low-rank ADI method (Penzl, 2000; Saak, 2009) is used which has proven to be an efficient algorithm for this goal and was recently subject to extensive research. It can be modified to deal with the special structure of the considered systems.

This paper is organized as follows: in the next section we briefly recall the main ideas behind balanced truncation for first order system. In Section 3 the second order systems of interest are transformed to equivalent first order systems and second order balanced truncation is introduced to regain the second order structure. The numerical solution of the occurring large-scale Lyapunov equation with the LR-ADI method is briefly discussed in Section 4. The performance of this model reduction approach for two test examples is shown in Section 5, and Section 6 concludes.

\section{BALANCED TRUNCATION FOR FIRST ORDER SYSTEMS}

Here we review the main workflow of balanced truncation for generalized state space systems

$$
\begin{aligned}
E \dot{z}(t) & =A z(t)+G u(t), \\
y(t) & =L z(t),
\end{aligned}
$$

where $E, A \in \mathbb{R}^{n \times n}, G \in \mathbb{R}^{n \times m}$ and $L \in \mathbb{R}^{p \times n}$. It is assumed that $E$ is nonsingular and that the spectrum $\Lambda(A, E)$ lies in the open left half plane $\mathbb{C}_{-}:=\{z \in$ $\mathbb{C}: \operatorname{Re}(z)<0\}$ such that $(3)$ is asymptotically stable. Note that balanced truncation originated for standard state space systems $\left(E=I_{n}\right)$ (Moore, 1981), but since systems of the form (3) are more important for our purpose, we follow the straightforward modification to generalized state space systems which was worked out in detail, e.g., in (Saak, 2009). The most important ingredients to carry out balanced truncation are the solutions of the generalized Lyapunov equations

$$
\begin{aligned}
& A P E^{T}+E P A^{T}=-G G^{T}, \\
& A^{T} Q E+E^{T} Q A=-L^{T} L .
\end{aligned}
$$

There, the symmetric matrices $P$ and $Q$ are the reachability and observability Gramian, respectively. The magnitude of the square roots of the eigenvalues of $P E^{T} Q E$ provides a joint measurement of how good certain states can be reached and observed. The values are system invariants and referred to as Hankel singular values. The main idea of balanced truncation is to identify and to truncate states which correspond to very small Hankel singular values, that is they are difficult to reach and to observe. Since both Gramians are positive semidefinite, we have Cholesky factorizations $P=R R^{T}$ and $Q=S S^{T}$. 
Using the singular value decomposition with decreasingly ordered singular values

$$
S^{T} E R=X \Sigma Y^{T}=\left[X_{1}, X_{2}\right] \operatorname{diag}\left(\Sigma_{1}, \Sigma_{2}\right)\left[Y_{1}, Y_{2}\right]^{T},
$$

where $\Sigma_{1} \in \mathbb{R}^{r \times r}$ contains the dominant singular values, the truncation matrices are constructed via

$$
T_{l}=\Sigma_{1}^{-\frac{1}{2}} X_{1}^{T} S^{T} \text { and } T_{r}=R Y_{1} \Sigma_{1}^{-\frac{1}{2}} .
$$

The reduced system of order $r$ is given by

$$
\begin{aligned}
\dot{\hat{z}}(t) & =\hat{A} \hat{z}(t)+\hat{G} u(t), \\
\hat{y}(t) & =\hat{L} \hat{z}(t),
\end{aligned}
$$

where $\hat{A}:=T_{l} A T_{r} \in \mathbb{R}^{r \times r}, \hat{G}:=T_{l} G \in \mathbb{R}^{r \times m}$ and $\hat{L}:=L T_{r} \in \mathbb{R}^{p \times r}$. Note that it can be shown that $T_{l} E T_{r}=$ $I_{r}$ holds (Saak, 2009). The stability of the original large system is preserved in the reduced ordel model. Moreover, one of the main advantages of balanced truncation is the available error bound

$$
\|y-\hat{y}\| \leq 2 \sum_{j=r+1}^{n} \sigma_{j}\|u\|,
$$

where the $\sigma_{j}$ are the singular values from the neglected block $\Sigma_{2}$. This error bound can be used to adaptively determine the order of the reduced system with respect to a prescribed error tolerance.

\section{BALANCED TRUNCATION FOR SYMMETRIC SECOND ORDER SYSTEMS}

The main focus of the article are second order systems (1), where $M, D, K$ are symmetric positive definite matrices and for $B, C_{\mathrm{p}}, C_{\mathrm{v}}$ we have one of the cases: either $B=C_{\mathrm{p}}^{T}, C_{\mathrm{v}}=0$ (case 1$)$ or $B=C_{\mathrm{v}}^{T}, C_{\mathrm{p}}=0$ (case 2$)$. The positive definiteness ensures that (1) is asymptotically stable.

\subsection{Reduction of Equivalent First Order Systems}

In order to follow the balanced truncation framework we have to transform the second order system into a first order system. The above assumptions on the matrices ensure that in both cases (1) can be transformed into a generalized state space system (3) with symmetric matrices $E, A \in \mathbb{R}^{2 n \times 2 n}$, with $G=L^{T} \in \mathbb{R}^{2 n \times m}$ and the augmented generalized state vector $z(t):=\left[x(t)^{T}, \dot{x}(t)^{T}\right]^{T}$. The matrices of the above generalized system are given by

$$
E:=\left[\begin{array}{cc}
D & M \\
M & 0
\end{array}\right], A:=\left[\begin{array}{cc}
-K & 0 \\
0 & M
\end{array}\right], G:=\left[\begin{array}{l}
B \\
0
\end{array}\right]
$$

for case 1 and

$$
E:=\left[\begin{array}{cc}
-K & 0 \\
0 & M
\end{array}\right], A:=\left[\begin{array}{cc}
0 & -K \\
-K & -D
\end{array}\right], G:=\left[\begin{array}{l}
0 \\
B
\end{array}\right]
$$

for case 2. This transformation is closely related to the rewriting of a quadratic eigenvalue problem into a generalized one, hence we may refer to it as linearization. In this context (6) relates to the first and (7) to the second companion linearization of a quadratic eigenvalue problem. It follows that these generalized systems are also asymptotically stable and that both generalized Lyapunov equations (4a) and (4b) are identical, such that $P=Q$ and consequently $R=S$. Hence, only the solution $P$, respectively its Cholesky factor $R$, of one generalized Lyapunov equation

$$
A P E+E P A=-G G^{T}
$$

is required. Since the solution of the Lyapunov equations (4) is the computationally most expensive task in balanced truncation, the symmetry of the chosen first order system leads to a reduction of the computational effort by half. We postpone the discussion about the actual numerical computation of $R$ until Section 4. Another consequence is that since $R^{T} E R$ is a symmetric matrix, its eigendecomposition can be used for building the truncation matrices $T_{l}$ and $T_{r}^{T}$. The matrices of the reduced order model will in general not have a block structure like the original matrices, such that the second order structure is lost. This can be cured by modifying balanced truncation as it is shown in the next section.

\subsection{Regaining the Second Order Structure}

Balanced truncation can be modified in order to generate a reduced order model which is also in second order form, see e.g., (Reis and Stykel., 2008). The main idea there is to partition the Gramians $P$ and $Q$ according to the structure present in in the equivalent generalized first order system:

$$
P=\left[\begin{array}{cc}
P_{\mathrm{p}} & P_{1,2} \\
P_{1,2}^{T} & P_{\mathrm{v}}
\end{array}\right], Q=\left[\begin{array}{cc}
Q_{\mathrm{p}} & Q_{1,2} \\
Q_{1,2}^{T} & Q_{\mathrm{v}}
\end{array}\right],
$$

where $P_{\mathrm{p}}, Q_{\mathrm{p}} \in \mathbb{R}^{n \times n}$ are called position, and $P_{\mathrm{v}}, Q_{\mathrm{v}} \in$ $\mathbb{R}^{n \times n}$ velocity reachability and observability Gramians, respectively. For our symmetric systems we only have to consider $P_{\mathrm{p}}$ and $P_{\mathrm{v}}$. Let $P_{\mathrm{p}}=R_{\mathrm{p}} R_{\mathrm{p}}^{T}, P_{\mathrm{v}}=R_{\mathrm{v}} R_{\mathrm{v}}^{T}$ be their Cholesky decompositions with nonsingular factors $R_{\mathrm{p}}, R_{\mathrm{v}} \in \mathbb{R}^{n \times n}$. Then one has four possible singular value decompositions

$$
\begin{aligned}
Z_{\alpha}^{T} M Z_{\beta} & =X_{\alpha \beta} \Sigma_{\alpha \beta} Y_{\alpha \beta}^{T} \\
& =\left[X_{\alpha \beta, 1}, X_{\alpha \beta, 2}\right] \operatorname{diag}\left(\Sigma_{\alpha \beta, 1}, \Sigma_{\alpha \beta, 2}\right)\left[Y_{\alpha \beta, 1}, Y_{\alpha \beta, 2}\right]^{T}
\end{aligned}
$$

with $\alpha, \beta \in\{\mathrm{p}, \mathrm{v}\}$. The $\Sigma_{\alpha \beta, 1} \in \mathbb{R}^{r \times r}$ contain the dominant singular values, and all other blocks have appropriate dimensions. Taking all possible choices for $\alpha, \beta$ into account leads to four different pairs of reduction matrices

$$
\begin{aligned}
& T_{r, \alpha \beta}:=R_{\alpha} Y_{\alpha \beta, 1} \Sigma_{\alpha \beta, 1}^{-\frac{1}{2}}, \\
& T_{l, \alpha \beta}:=R_{\beta} X_{\alpha \beta, 1} \Sigma_{\alpha \beta, 1}^{-\frac{1}{2}} .
\end{aligned}
$$

For $\alpha=\beta=\mathrm{p}$ the approach is called position-position (PP), for $\alpha=\beta=\mathrm{v}$ velocity-velocity (VV), for $\alpha=\mathrm{v}$, $\beta=\mathrm{p}$ velocity-position (VP), and for $\alpha=\mathrm{p}, \beta=\mathrm{v}$ position-velocity (PV) second order balanced truncation, respectively. All four pairs of these transformation matrices are summarized in Table 1. Hence, there are in total four different reduced order models in second order form

$$
\begin{aligned}
\hat{M}_{\alpha \beta} \ddot{\hat{x}}(t)+\hat{D}_{\alpha \beta} \dot{\hat{x}}(t)+\hat{K}_{\alpha \beta} \hat{x}(t) & =\hat{B}_{\alpha \beta} u(t), \\
\hat{y}(t) & =\hat{C}_{\alpha \beta}^{\mathrm{p}} \hat{x}(t)+\hat{C}_{\alpha \beta}^{\mathrm{v}} \dot{\hat{x}}(t)
\end{aligned}
$$

Table 1. Left and right transformations matrices of balanced truncation for second order systems.

\begin{tabular}{|c|c|c|}
\hline Type & right transformation & left transformation \\
\hline \hline $\mathrm{PP}$ & $T_{r, \mathrm{pp}}:=R_{\mathrm{p}} Y_{\mathrm{pp}, 1} \Sigma_{\mathrm{pp}, 1}^{-\frac{1}{2}}$ & $T_{l, \mathrm{pp}}:=R_{\mathrm{p}} X_{\mathrm{pp}, 1} \Sigma_{p p, 1}^{-\frac{1}{2}}$ \\
$\mathrm{PV}$ & $T_{r, \mathrm{pv}}:=R_{\mathrm{p}} Y_{\mathrm{pv}, 1} \Sigma_{\mathrm{pv}, 1}^{-\frac{1}{2}}$ & $T_{l, \mathrm{pv}}:=R_{\mathrm{v}} X_{\mathrm{pv}, 1} \Sigma_{\mathrm{p}}^{-\frac{1}{2}}$ \\
$\mathrm{VP}$ & $T_{r, \mathrm{vp}}:=R_{\mathrm{v}} Y_{\mathrm{vp}, 1} \Sigma_{\mathrm{vp}, 1}^{-\frac{1}{2}}$ & $T_{l, \mathrm{vp}}:=R_{\mathrm{p}} X_{\mathrm{vp}, 1} \Sigma_{\mathrm{v}}^{-\frac{1}{2}}$ \\
$\mathrm{PV}$ & $T_{r, \mathrm{vv}}:=R_{\mathrm{v}} Y_{\mathrm{vv}, 1} \Sigma_{\mathrm{vv}, 1}^{-\frac{1}{2}}$ & $T_{l, \mathrm{vv}}:=R_{\mathrm{v}} X_{\mathrm{vv}, 1} \Sigma_{\mathrm{v}}^{-\frac{1}{2}}$ \\
\hline
\end{tabular}


with

$$
\begin{aligned}
\hat{M}_{\alpha \beta} & :=T_{l, \alpha \beta}^{T} M T_{r, \alpha \beta} \in \mathbb{R}^{r \times r}, \\
\hat{D}_{\alpha \beta} & :=T_{l, \alpha \beta}^{T} D T_{r, \alpha \beta} \in \mathbb{R}^{r \times r}, \\
\hat{K}_{\alpha \beta} & :=T_{l, \alpha \beta}^{T} K T_{r, \alpha \beta} \in \mathbb{R}^{r \times r}, \\
\hat{B}_{\alpha \beta} & :=T_{l, \alpha \beta}^{T} B \in \mathbb{R}^{r \times m}, \\
\hat{C}_{\alpha \beta}^{\mathrm{p}} & :=C^{\mathrm{p}} T_{r, \alpha \beta} \mathbb{R}^{m \times r}, \\
\hat{C}_{\alpha \beta}^{\mathrm{v}} & :=C^{\mathrm{v}} T_{r, \alpha \beta} \in \mathbb{R}^{m \times r} .
\end{aligned}
$$

For this type of balanced truncation, there is in general no error bound similar to (5). For an adaptive determination of the reduced order model, one can, e.g., monitor the ratio of the entries in $\Sigma_{\alpha \beta}$

$$
\frac{\sigma_{j, \alpha \beta}}{\sigma_{1, \alpha \beta}} \leq \epsilon .
$$

Moreover, stability is not preserved for general systems using the approach in (Reis and Stykel., 2008). We now show that for the symmetric systems it can be preserved for two particular choices from (10). Since $M$ is symmetric positive definite, so are $R_{\mathrm{p}}^{T} M R_{\mathrm{p}}$ and $R_{\mathrm{v}}^{T} M R_{\mathrm{v}}$ and hence, because now their singular value decomposition coincides with their eigendecomposition, $X_{\mathrm{pp}}=Y_{\mathrm{pp}}$ and $X_{\mathrm{vv}}=Y_{\mathrm{vv}}$. Consequently, $T_{r, \mathrm{pp}}=T_{l, \mathrm{pp}}$ and $T_{r, \mathrm{vv}}=T_{l, \mathrm{vv}}$ holds such that position-position and velocity-velocity second order balanced truncation use one-sided transformations. Therefore, the reduced mass, damping and stiffness matrices remain symmetric positive definite such that the reduced order models are asymptotically stable. Note that the reduced output is still the reduced transposed input matrix in these two approaches.

Beyond that, since $R_{\mathrm{v}}^{T} M R_{\mathrm{p}}=\left(R_{\mathrm{p}}^{T} M R_{\mathrm{v}}\right)^{T}$ we have $X_{\mathrm{pv}}=$ $Y_{\mathrm{vp}}, X_{\mathrm{vp}}=Y_{\mathrm{pv}}$ which leads to $T_{r, \mathrm{pv}}=T_{l, \mathrm{vp}}$ and $T_{r, \mathrm{vp}}=$ $T_{l, \mathrm{pv}}$. One easily sees then that the mass, damping and stiffness matrices of the position-velocity reduced order model are the transposes of the matrices of the velocityposition reduced order model. Additionally, it holds for case 1 that $\hat{B}_{\mathrm{pv}}=\left(\hat{C}_{\mathrm{vp}}^{\mathrm{p}}\right)^{T}$ and $\hat{B}_{\mathrm{vp}}=\left(\hat{C}_{\mathrm{pv}}^{\mathrm{p}}\right)^{T}$. For case 2 similar relations involving $\hat{C}^{\mathrm{v}}$ exist. The position-velocity is the adjoint of the velocity-position reduced order model such that the spectral- and Frobenius norm of the transfer functions of both systems are identical as it can be seen in the frequency response plots for the examples in Section 5 .

\section{SOLUTION OF THE LYAPUNOV EQUATION}

Since for our purposes, $M, D, K$ and thus $E$ and $A$ will be large and sparse matrices, classical solution strategies for (8) involving the eigendecomposition of $(A, E)$ cannot be applied due to their cubic complexity. We therefore approximate the Cholesky factor $R$ by a low-rank solution factor $\hat{R} \in \mathbb{R}^{n \times d}, d \ll n$ such that $\hat{R} \hat{R}^{T} \approx P$. For this we use the generalized low-rank alternating direction implicit method (G-LR-ADI) (Penzl, 2000; Saak, 2009; Benner and Saak, 2011) which follows for $j=1, \ldots, j_{\max }$ the iteration

$$
\begin{aligned}
& V_{1}=\sqrt{-2 \operatorname{Re}\left(\mu_{1}\right)}\left(A+\mu_{1} E\right)^{-1} G, \\
& V_{j}=\sqrt{\frac{\operatorname{Re}\left(\mu_{j}\right)}{\operatorname{Re}\left(\mu_{j-1}\right)}}\left(I-\left(\mu_{j}+\overline{\mu_{j-1}}\right)\left(A+\mu_{j} E\right)^{-1}\right) E V_{j},
\end{aligned}
$$

with $\hat{R}=\left[V_{1}, \ldots, V_{j_{\max }}\right]$. There the $\mu_{j} \in \mathbb{C}_{-}$are shift parameters steering the convergence. In a large-scale setting they are usually obtained from a small number of Ritz values of $E^{-1} A$ solving approximately a rational min-max problem (Penzl, 2000). Since systems of the form (1) come often from a spacial finite element discretization of elastic mechanical bodies, the matrix pair $(A, E)$ of $(6),(7)$, and equivalently the quadratic matrix polynomial associated to (1), will most likely have complex eigenvalues. Hence the Ritz values as well as the shift parameters might be complex numbers. In the presence of such complex shift parameters, the iteration (12) will consequently produce a complex low-rank factor $\hat{R}$. This is undesirable from a numerical point of view since complex arithmetic operations are more expensive than real ones. Moreover, a complex low-rank factor $\hat{R}$ will also make the transformation matrices $(9)$ and hence the matrices defining the reduced order model (10) complex. However, if the both $\mu_{j}, \mu_{j+1}:=\overline{\mu_{j}}$ are consecutive shift parameters this can be circumvented by following the approach in (Benner et al., 2011) which allows the computation of real low-rank factors even if complex shift parameters are used. The main result there is that the iterate with respect to $\mu_{j+1}$ can be constructed by

$$
V_{j+1}=\operatorname{Re}\left(V_{j}\right)-\jmath \operatorname{Im}\left(V_{j}\right)+2 \frac{\operatorname{Re}\left(\mu_{j}\right)}{\operatorname{Im}\left(\mu_{j}\right)} \operatorname{Im}\left(V_{j}\right)
$$

such that the solution of the complex linear system with $\overline{\mu_{j}}$ is not required. Thus the amount of complex arithmetic operations and storage is greatly decreased.

For reasons of efficiency, instead of working with the augmented matrices $E, A, G$ and the associated linear systems

$$
(A+\mu E) \hat{V}=E V
$$

of dimension $2 n \times 2 n$ the iteration (12) can be rewritten such that linear systems of the form

$$
\left(\mu^{2} M-\mu D+K\right) \hat{V}^{(1)}=\hat{W}
$$

of dimension $n \times n$ have to be solved (Benner and Saak, 2011) which involve the original matrices $M, D, K, B$. This modification is usually referred to as second order LRADI (SO-LR-ADI). Altogether this leads for case 1 to the second order LR-ADI (SO-LR-ADI) given in Algorithm 1. The iteration for case 2 is similar and can be obtained from using the augmented matrices (7) and rewriting the associated iteration (12).

\section{NUMERICAL EXAMPLES}

We investigate the quality of the model reduction approach using two test systems which both represent finite element models of elastic structures and belong to case 1 . The first model represents a Bernoulli beam, where the discretization leads to a second order system of dimension $n=3000$ with $m=1$. The second system was obtained from a discretization of a secondary deformable mirror which is part of a telescope. There, $n=83508$ and we take the first $m=5$ of the original 672 columns in $B$. The generalized Lyapunov equations of dimension 6000 and 167016 were solved with SO-LR-ADI (Algorithm 1) until the norm of the normalized Lyapunov residuals dropped below $10^{-8}$ which required 76 and 88 iterations of SOLR-ADI, respectively. Second order balanced truncation was applied to obtain the reduced order models, where in both examples the dimensions of the reduced systems was 


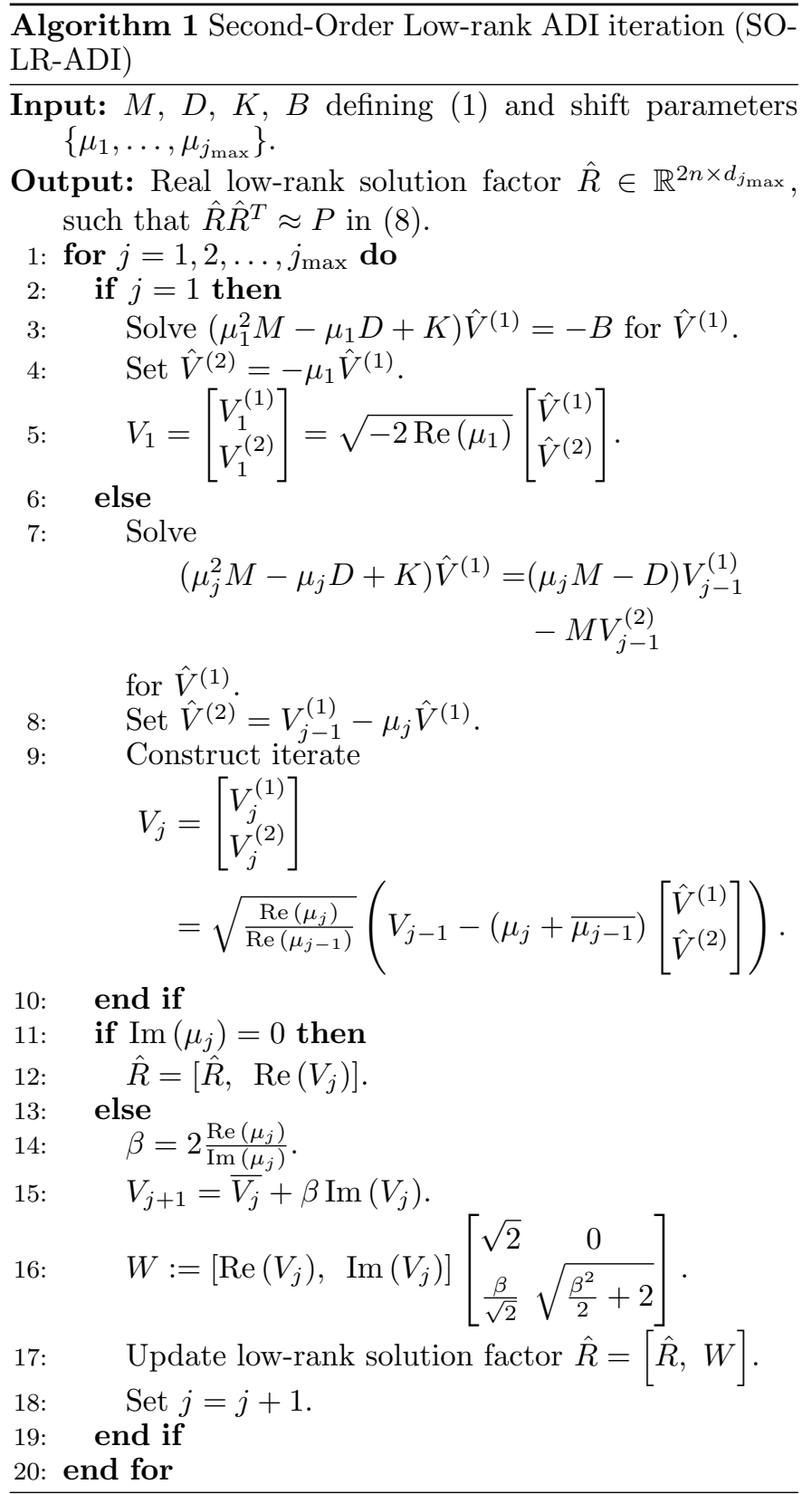

determined using the ratio (11) and a truncation tolerance $\epsilon=10^{-10}$. Additionally, the reduction to first order systems with standard balanced truncation (in the sequel denoted by $\mathrm{BT}_{1}$ ) was carried out using both (11) and the error bound (5) $\left(\mathrm{BT}_{2}\right)$ to set the reduced dimension. We measure the accuracy of the different reduction approaches using the transfer functions

$$
\begin{aligned}
H(s) & :=C\left(s^{2} M+s D+K\right)^{-1} B, \\
\hat{H}(s) & :=\hat{C}\left(s^{2} \hat{M}+s \hat{D}+\hat{K}\right)^{-1} \hat{B}, \\
\hat{F}(s) & :=\hat{L}(s \hat{E}-\hat{A})^{-1} \hat{G}
\end{aligned}
$$

with $s=\mathrm{i} \omega, \omega \in \mathbb{R}$ of original and reduced order systems, as well as the associated relative errors

$$
\begin{aligned}
\epsilon_{\mathrm{rel}} & =\|H(s)-\hat{H}(s)\|_{2} /\|H(s)\|_{2}, \\
\text { or } \epsilon_{\mathrm{rel}} & =\|H(s)-\hat{F}(s)\|_{2} /\|H(s)\|_{2},
\end{aligned}
$$

where the quantities involving $\hat{F}$ are used for the reduction to a generalized first order system $\left(\mathrm{BT}_{1}\right.$ and $\left.\mathrm{BT}_{2}\right)$. The frequency parameter $\omega$ varies through logarithmically spaced points from the interval $\left[1,10^{4}\right] \mathrm{Hz}$ for the beam example and $\left[10^{-3}, 5 \cdot 10^{3}\right] \mathrm{Hz}$ for the mirror system.

Table 2 shows the dimensions of the obtained reduced order models and the maximal relative error in the considered frequency range for both examples. Fig. 1 and 3 show the frequency response in terms of the spectral norm of exact and reduced transfer functions for both examples. Apparently, there is no distinguishable difference between the frequency response plots of original and reduced models. In Fig. 2 and 4 the associated relative errors along the considered frequency interval is plotted showing that the accuracy of the models obtained with second order and standard balanced truncation using the singular value ratio (11) is very high. The relative error of standard balanced truncation using the error bound (5) is much higher as it is also visible from the values in Table 2 . This leads to the conclusion that monitoring the relative singular value decays yields more accurate results for these systems. Similar experiments (not reported here) using a fixed dimension of the reduced order model instead of using (5) or (11) also showed a higher accuracy obtained with second order balanced truncation. The equivalence of the frequency response in the spectral norm of positionvelocity and velocity-position reduced order models is also clearly visible by the relative error plots.

Table 2. Dimensions of the reduced systems and maximal relative error for both examples.

\begin{tabular}{|c||c|c|c|c|}
\hline \multicolumn{1}{|c||}{} & \multicolumn{2}{c|}{ beam } & \multicolumn{2}{c|}{ mirror } \\
\hline Type & $r$ & $\max \left(\epsilon_{\mathrm{rel}}\right)$ & $r$ & $\max \left(\epsilon_{\mathrm{rel}}\right)$ \\
\hline $\mathrm{PP}$ & 12 & $2.8 \cdot 10^{-8}$ & 60 & $1.1 \cdot 10^{-5}$ \\
$\mathrm{VV}$ & 27 & $4.7 \cdot 10^{-10}$ & 105 & $4.3 \cdot 10^{-8}$ \\
$\mathrm{PV}$ & 19 & $3.2 \cdot 10^{-9}$ & 98 & $4.2 \cdot 10^{-8}$ \\
$\mathrm{VP}$ & 19 & $3.2 \cdot 10^{-9}$ & 98 & $5.4 \cdot 10^{-8}$ \\
\hline $\mathrm{BT}_{1}$ & 24 & $3.9 \cdot 10^{-9}$ & 132 & $5.0 \cdot 10^{-8}$ \\
$\mathrm{BT}_{2}$ & 9 & $1.5 \cdot 10^{-3}$ & 68 & $3.7 \cdot 10^{-4}$ \\
\hline
\end{tabular}

\section{CONCLUSIONS}

We have investigated second order balanced truncation for the special class of symmetric second order systems. This structure is beneficial, since it enables the preserving stability of the dynamical system in the reduction process

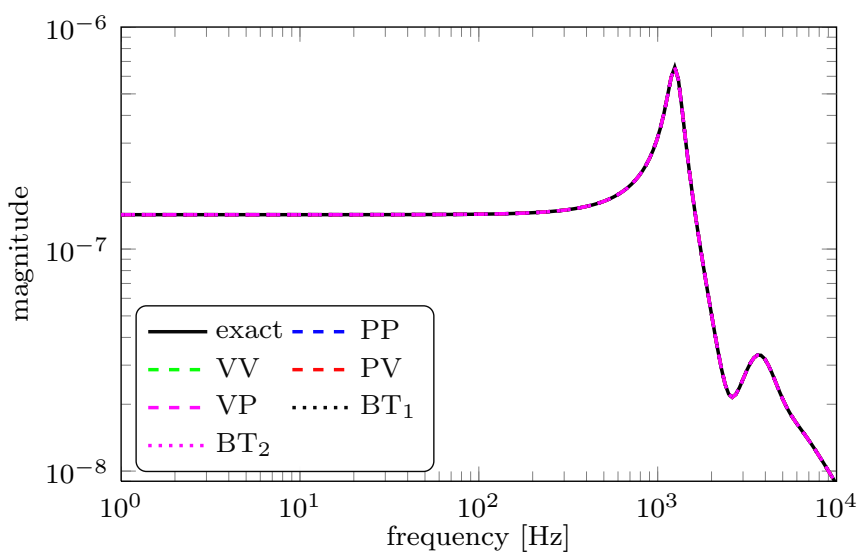

Fig. 1. Bode plots of exact and reduced systems for the beam model. 


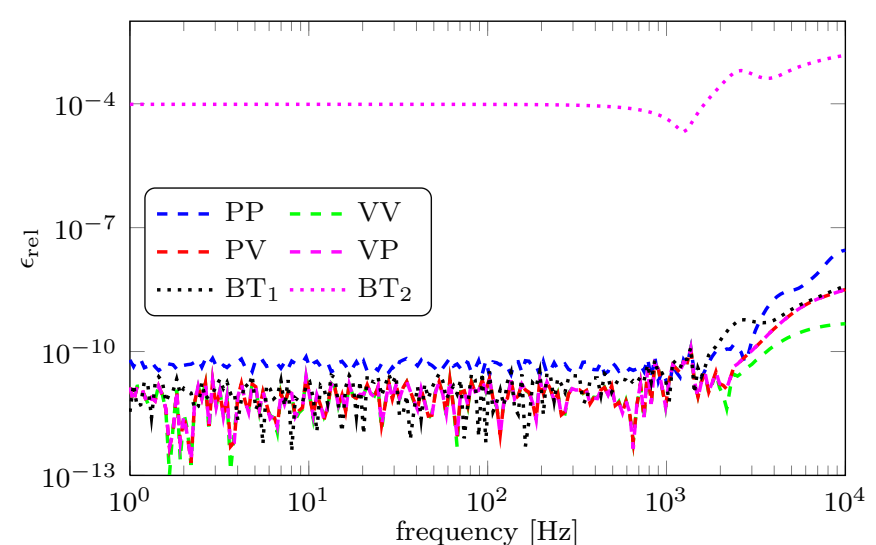

Fig. 2. Relative error of the reduced systems for the beam model.

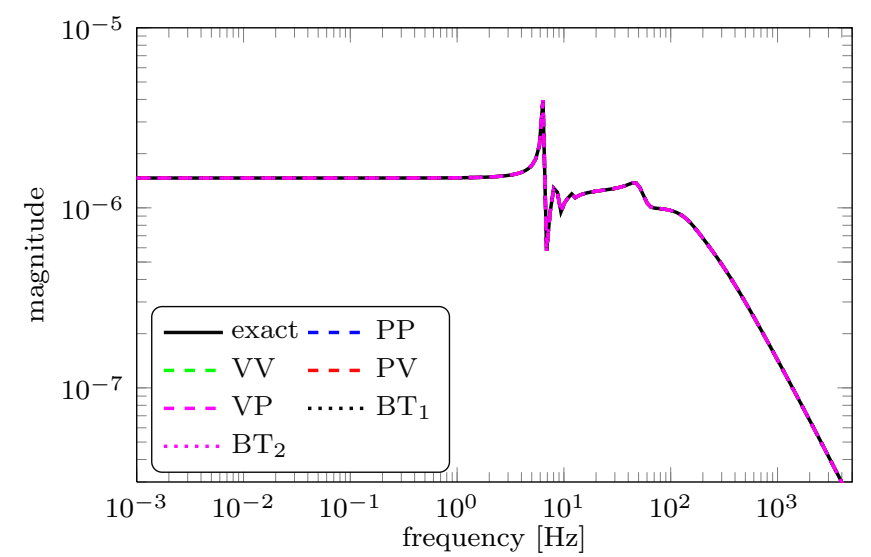

Fig. 3. Bode plots of exact and reduced systems for the mirror model.

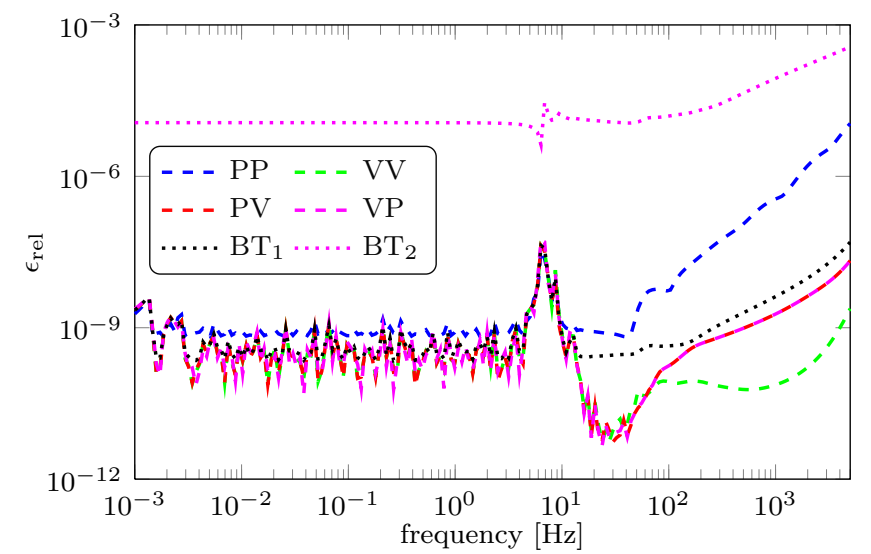

Fig. 4. Relative error of the reduced systems for the mirror model.

and it also greatly reduces the amount of required computational work. The occurring generalized large-scale Lyapunov equations were efficiently solved with an adequately adapted version of the low-rank ADI method. Numerical experiments using finite element discretizations of real elastic bodies confirmed a high accuracy of the obtained reduced order models, which also lead to more accurate results than following the standard balanced truncation approach by reducing the second order system to a reduced first order state space system.

\section{ACKNOWLEDGEMENTS}

The authors thank Christine Nowakowski from the Institute of Engineering and Computational Mechanics and Thomas Ruppel from the Institute for System Dynamics, both at the University of Stuttgart, for providing the Bernoulli beam and mirror example, respectively.

\section{REFERENCES}

Benner, P., Kürschner, P., and Saak, J. (2011). Efficient handling of complex shift parameters in the low-rank Cholesky factor ADI method. Technical Report MPIMD/11-08, Max Planck Institute Magdeburg Preprints.

Benner, P. and Saak, J. (2011). Efficient Balancing based MOR for Large Scale Second Order Systems. Math. Comput. Model. Dyn. Sys., 17(2), 123-143. DOI:10.1080/13873954.2010.540822.

Moore, B.C. (1981). Principal component analysis in linear systems: controllability, observability, and model reduction. IEEE Trans. Automat. Control, AC-26(1), $17-32$.

Penzl, T. (2000). A cyclic low rank Smith method for large sparse Lyapunov equations. SIAM J. Sci. Comput., 21(4), 1401-1418.

Reis, T. and Stykel., T. (2008). Balanced truncation model reduction of second-order systems. Math. Comput. Model. Dyn. Syst., 14(5), 391-406.

Saak, J. (2009). Efficient Numerical Solution of Large Scale Algebraic Matrix Equations in PDE Control and Model Order Reduction. Ph.D. thesis, TU Chemnitz. Available from http://nbnresolving.de/urn:nbn:de:bsz:ch1-200901642. 